\title{
Conceito de Saúde e Intersetorialidade: Implicações no Cotidiano da Atenção Primária à Saúde
}

\section{The Concept of Health and Intersectoriality: Implications on everyday Primary Health care Concepto de Salud e Intersectorialidad: Implicaciones Diarias de la Atención Primaria de Salud}

\author{
Edna Mara Mendonça ${ }^{1}$ \\ Instituto René Rachou - Fiocruz Minas \\ Fernanda Moura Lanza \\ Universidade Federal de São João del-Rei (UFSJ)
}

\begin{abstract}
Resumo
Buscou-se compreender as noções de saúde e intersetorialidade pelos profissionais da Atenção Primária à Saúde e verificar suas implicações no cotidiano. Para tal, desenvolveu-se uma pesquisa qualitativa, ancorada no interacionismo simbólico, em um município de Minas Gerais, entre fevereiro e julho de 2018. A coleta de dados foi realizada por meio de entrevistas semiestruturadas realizadas com 59 profissionais da Secretaria Municipal de Saúde. Os resultados apontam que os profissionais que possuem o conceito ampliado de saúde como objeto de seu trabalho realizam as práticas com outras políticas públicas; já o modelo biomédico reduz a atuação ao tratamento de doenças que restringem a atuação profissional à equipe ou à rede intrassetorial. A intersetorialidade surge como estratégia de cuidado em rede, pois prioriza a integração de diferentes setores para a resolução de problemas comuns. Os resultados apontam para a necessidade de aprofundar a discussão do objeto de trabalho em saúde. Palavras-chave: saúde global, Sistema Único de Saúde, políticas públicas de saúde, atenção primária à saúde, colaboração intersetorial
\end{abstract}

\begin{abstract}
This article aims at understanding the ideas of health and intersectoriality by Primary Health Care employees and verifies their implications. To do so, a qualitative research, supported by symbolic interactionism, was developed in a municipality of Minas Gerais from February to July 2018.

Semi-structured interviews with 59 employees of the Municipal Health Department were carried out. The results show that workers who absorb the concept of health expansion spread it to other public policies and/or communities' partnerships. Moreover, the biomedical model limits the performance to curative attention, restricting the employee's performance in their teams or even in intrasectoral network. The idea of intersectoriality emerges as a network care strategy since it prioritizes the integration of several sectors to solve common issues. Thus, this article may contribute to a better understanding of the work in health. Keywords: global health, Brazilian National Health Service, public health policies, primary health care, intersectoral collaboration
\end{abstract}

\section{Resumen}

Intentamos comprender las nociones de salud e intersectorialidad de los profesionales de Atención Primaria de Salud y verificar sus implicaciones en la vida diaria. Con este fin, se realizó una investigación cualitativa, anclada en el interaccionismo simbólico, en un municipio de Minas Gerais, entre febrero y julio de 2018. La recopilación de datos se realizó a través de entrevistas semiestructuradas realizadas con 59 profesionales de la Secretaría Municipal de Salud. Los resultados indican que los profesionales que tienen el concepto amplio de salud como su objeto de trabajo realizan prácticas con otras políticas públicas; el modelo biomédico, por otro lado, reduce el desempeño para el tratamiento de enfermedades que restringen el desempeño profesional al equipo o la red intrasectorial. La intersectorialidad surge como una estrategia de atención en red, ya que prioriza la integración de diferentes sectores para resolver problemas comunes. Los resultados apuntan a la necesidad de profundizar la discusión del objeto de trabajo en salud.

Palabras clave: salud global, Sistema Único de Salud, políticas de salud pública, atención primaria de salud, colaboración intersectorial

\footnotetext{
${ }^{1}$ Endereço de contato: Rua Lagoa da Prata, 276, Bairro Santo Antônio, Pará de Minas, Minas Gerais. CEP: 35661-183. Telefones: 37 99150-0097. E-mail: ednamaras@gmail.com
} 


\section{Introdução}

A Conferência Internacional de Alma Ata (1978) trouxe nova proposta de cuidados em saúde, com a mudança na concepção de saúde, que passou a ser vista de maneira mais ampliada e como fenômeno de múltiplas determinações. Isso exigiu a transformação dos serviços de saúde na busca pela integralidade no cuidado, e a constituição de uma rede de práticas intersetoriais tornou-se norte para articulações inovadoras que correspondessem à nova concepção (Molina, 2018).

Estudos recentes apontam a importância da prática intersetorial no campo da saúde, dada a magnitude dos determinantes sociais, o que torna difícil para uma instituição resolver os problemas em um único setor (Dubois, St-Pierre, \& Veras, 2015; Fisher et al., 2017). A união entre dispositivos institucionais, não institucionais e comunidade pode favorecer o atendimento integral, a efetivação do Sistema Único de Saúde (SUS), além da inclusão social, o que nos leva à apreensão dos processos de colaboração intersetorial para avançar a outras formas de integração de organização das políticas públicas (Lotta \& Favareto, 2016).

Na 70a Assembleia Geral das Nações Unidas, em setembro de 2015, a saúde foi posta como um dos Objetivos do Desenvolvimento Sustentável (ODS) - sendo incorporada entre as 17 dimensões - e colocou-se, para seus atores, o desafio de inserir-se em uma agenda intersetorial (para além da saúde), capaz de estimular o enfrentamento dos seus determinantes sociais em territórios, o que dialoga com o desenvolvimento sustentável. Os ODS apareceram, então, como "Nova Agenda Universal" ou Agenda 2030, o que nos faz presumir a ocorrência de discussões nos próximos anos, principalmente quando relacionadas à saúde e qualidade de vida (United Nations, 2015).

No Brasil, os serviços de Atenção Primária à Saúde (APS), que são responsáveis pela coordenação do cuidado na Rede de Atenção à Saúde (RAS), vem assumindo missão estratégica para a organização e ampliação da efetividade dos sistemas de saúde (Almeida et al., 2018). A expansão da Estratégia Saúde da Família (ESF) e, posteriormente, a implantação dos Núcleos de Apoio a Saúde da Família (NASF), hoje conhecidos como Núcleo Ampliado da Saúde da Família e Atenção Básica (NASF-AB), tiveram como missão o fortalecimento da APS, com incorporação de novas práticas e mudanças de paradigmas de cuidado, diminuição das iniquidades sociais e aproximação com a comunidade (Tesser, 2017).

Nesse sentido, atores dos dispositivos da ESF e NASF-AB apostam na saúde como produção da vida, e são utilizadas estratégias para tessitura de uma rede de serviços, de ações de cuidado, de pessoas, provocando encontros, acontecimentos (Yasui, Luzio, \& Amarante, 2018). A saúde é, portanto, o objeto de trabalho dos profissionais que deve ser mantido em constante reflexão para que suas práticas se aproximem da finalidade da APS disposta na Política Nacional de Atenção Básica. No entanto a saúde tem duas vertentes; há quem a considere como um emaranhado de fatores biológicos e outros como um fenômeno complexo e com múltiplas determinações. Ambas as visões implicam a forma como vivemos e entendemos as condições de saúde das populações (Barreto, 2017). Percebe-se que a forma como os trabalhadores e gestores entendem o objeto de trabalho no cotidiano dos serviços de saúde da APS refletem na maneira desses profissionais em organizar o cuidado (Souza et al., 2010).

Nesse contexto, questiona-se: quais as concepções de saúde surgem no cotidiano dos profissionais da ESF, NASF-AB e gestores? Qual significado os profissionais da ESF, NASF-AB e gestores 
atribuem à intersetorialidade? Quais as implicações de ações intersetoriais no cotidiano do cuidado na APS?

Assim, este estudo teve como objetivos: compreender as noções de saúde e intersetorialidade dos profissionais nas ESF, NASF-AB e gestores; e verificar as possíveis implicações de ações intersetoriais para o cuidado, pois partimos do pressuposto de que a promoção da saúde é um trabalho que se faz e refaz como efeito de um coletivo, de acordo com seus interesses pessoais e interações sociais.

\section{Metodologia}

Pesquisa de abordagem qualitativa, a qual se utilizou do referencial teórico do Interacionismo Simbólico para suporte à análise do objeto da pesquisa (Blumer, 1969).

O cenário da pesquisa foi constituído por cinco unidades de ESF e quatro equipes de NASF-AB de um município de médio porte do Estado de Minas Gerais que possui 93\% da população coberta pela ESF. Essas equipes foram sorteadas aleatoriamente, sem definição dos critérios de inclusão e exclusão, sendo que o número de equipes inseridas no estudo foi definido posteriormente, mediante a saturação dos dados coletados.

A partir do cenário, deu-se a escolha dos participantes. Os critérios de inclusão dos participantes tiveram como base todos os membros da respectiva ESF ou NASF-AB do município que estavam em atuação há mais de um ano no serviço. Os critérios de exclusão do estudo foram o profissional estar de férias ou qualquer outro motivo de afastamento no período da coleta de dados. Foram também convidados a participar do estudo os gestores da Secretaria Municipal de Saúde (SMS).

O teste-piloto foi realizado em outro município, com serviços de saúde com características semelhantes ao do estudo, para avaliar a adequação do instrumento e a qualidade da coleta de dados.

As entrevistas foram realizadas pela pesquisadora principal, ocorreram no período de fevereiro a julho de 2018 e tiveram a participação de 59 colaboradores, sendo: (a) 36 profissionais da ESF, entre estes, dois médicos, cinco enfermeiros, oito auxiliares/técnicos de enfermagem, 17 agentes comunitários de saúde, dois cirurgiões-dentistas e dois auxiliares em saúde bucal; (b) 20 trabalhadores do NASF-AB, sendo dois psicólogos, um assistente social, quatro fisioterapeutas, quatro nutricionistas, dois professores de educação física, três fonoaudiólogos, três farmacêuticos e um enfermeiro com formação em Práticas Integrativas e Complementares (PICS); (c) três gestores da Secretaria Municipal de Saúde. Quanto às pessoas que não atenderam aos critérios de inclusão, foram 29 em sua totalidade. Destas, 20 (69\%) foram das ESF e 9 (31\%) do NASF-AB. Apenas 1 (3,5\%) negou-se a participar da pesquisa, sem justificativa alguma.

As entrevistas individuais foram agendadas mediante aceite do profissional, após a explicação do objetivo do estudo, seus riscos e benefícios, de acordo com a disponibilidade dos participantes, no próprio local de trabalho, em uma sala reservada para sigilo das informações e para evitar interrupções. As entrevistas foram gravadas por meio de um dispositivo móvel (iPhone) e duraram, em média, 8 minutos e 20 segundos. Não houve necessidade de repetição de nenhuma entrevista.

A coleta de dados foi realizada de acordo com as etapas: contato com o campo e apresentação da proposta, realização das entrevistas individuais e elaboração do diário de cam- 
po. A coleta terminou quando houve saturação das evidências, ou seja, quando houve reincidência das respostas, mas sem desprezar conteúdos importantes para o estudo (Minayo, 2017).

O conteúdo das entrevistas individuais foi transcrito na íntegra de forma criteriosa. Medidas de proteção ao anonimato das informações referentes às entrevistas individuais foram tomadas. O sigilo foi mantido por meio da adoção da letra E (Entrevistado), seguida de número sequencial às entrevistas realizadas subsequentes (E1, E2. . .). As transcrições não foram devolvidas aos participantes para comentários e/ou correções. Após essa etapa, a pesquisadora principal realizou a análise de conteúdo (Bardin, 2011). Procedeu-se a leitura flutuante que favoreceu a identificação dos núcleos de sentidos emergidos no conteúdo das falas. A exploração do material consistiu na codificação e categorização dos dados obtidos. O presente artigo apresenta os resultados da categoria empírica, que são as concepções de saúde e a manifestação de ações no cotidiano da APS: intrassetorialidade versus intersetorialidade.

Pesquisa aprovada no Comitê de Ética em Pesquisa Envolvendo Seres Humanos, sob CAAE 79985917.9.0000.5545, parecer 2.469.057.

\section{Resultados e Discussão}

Todos os participantes da pesquisa expuseram suas concepções de saúde que refletiram diferentes dimensões conceituais: "A saúde é a ausência de doença e você cuidar dela" (E26 - ESF). "Saúde para mim é lazer, cultura, educação, esporte" (E15 - ESF).

A Reforma Sanitária Brasileira e a consequente implantação do SUS na Constituição da República Federativa Brasileira de 1988 possibilitaram a ampliação do conceito de saúde, colocando doença e saúde em constante relação. A saúde passou a ser colocada como " $a$ potência para lidar com a existência" e a doença como "sentimento de vida contrariada". Assim, os sentidos da saúde e da doença passaram a ter caráter subjetivo e relacionado ao modo de vida individual ou coletivo, podendo ser ainda englobados social, histórica e culturalmente (Czeresnia, Maciel, \& Oviedo, 2013).

No modelo biomédico tradicional, a pessoa é vista como uma doença a ser tratada. Assim, os profissionais optam por realizar suas ações de maneira isolada e/ou centralizada em sua especificidade, ou, então, sua atuação se dá meramente na rede de saúde, a chamada rede intrassetorial (Barreto, 2017; Menezes, Scherer, Verdi, \& Pires, 2017). A aproximação com o modelo biomédico foi constatada por atores da ESF: "Saúde compreende um bem-estar físico e emocional. É uma pergunta complexa, não é? Eu entendo como isso (E18 - ESF); "Saúde... É estar bem com você mesmo. Equilibrado. Não ter nada doendo em você (E36-ESF).

A manutenção desse tipo de prática individualiza em detrimento de questões que são coletivas, de cunho social, leva a rede a funcionar de maneira restrita e setorializada, não privilegiando os encontros coletivos com a complementaridade de práticas. A consolidação de um sistema de saúde ancorado no conhecimento biomédico é incapaz de intervir sobre os determinantes do processo saúde-doença. Contudo, na literatura, questiona-se a articulação entre a rede de serviços quando esta é reduzida a encaminhamentos para diagnósticos e outras especialidades, visto que esses raramente se desdobram em outras ações que impactam os determinantes sociais da saúde (Barreto, 2017; Menezes et al., 2017). 
E é por isso que a reorientação das práticas de saúde aponta não apenas para a necessidade de construir novas instrumentalidades, mas também a construção de novas concepções de cuidado. O profissional deve ter um olhar amplo, ser comprometido com a prática de trabalho em equipe e integrado com disponibilidade para exercer um fazer coletivo com corresponsabilização e acolhimento das reais necessidades da população (Garbois, Sodré, \& Dalbello-Araujo, 2017; Fertonani, 2015).

Saúde é um conjunto, não pode ser definida apenas como bem-estar físico. Além do bem-estar físico, você tem o social, você tem o educacional [. . .]. Então acho que o conjunto de ações, não é?! Quanto mais ações, quanto mais esclarecida a população, mais saudável, a gente vai conseguir desse pessoal mais saúde. Eu acho que hoje em dia, se você foca só numa área, você deixa várias outras descobertas, e acaba que isso vai gerar a longo prazo algum problema na sua população, e que vai acarretar bem possivelmente alguma deficiência na sua área de saúde. (E12 - NASF-AB)

Alguns países europeus, o Canadá e a Austrália têm realizado ações públicas com vistas à superação desse dilema, com a implementação da abordagem "Saúde em Todas as Políticas" ou "Health in All Policies (HiAP)". Os fundamentos teóricos baseiam-se na ideia de que a saúde é fortemente determinada socialmente, e sua ação envolve a responsabilidade de todo o governo. Essa estrutura de política faz uso da colaboração intersetorial para melhorar a saúde e a equidade na saúde das comunidades (Shankardass et al., 2018; Matting, Cantoreggi, Simos, Kruit, \& Christie, 2017). A perspectiva internacional dialoga com algumas falas que vão ao encontro do conceito ampliado de saúde ou processo saúde-doença, como:

Agora o fazer saúde, é olhar muito para a diversidade do público que a gente atende. Não focar apenas na queixa que aquele paciente traz, e sim olhá-lo num todo. O contexto e os hábitos de vida desse paciente são fatores determinantes para que a gente consiga realmente fazer saúde em qualquer instância. (E21 - NASF-AB)

É um conjunto de situações que envolvem bem-estar, acomodação, moradia, alimentação, é um grupo de coisas envolvidas na saúde. (E53 - ESF)

Saúde eu acho que é um conceito muito complexo, que vai muito além da ausência da doença, não é?! Saúde é a capacidade da pessoa exercer seus direitos e seus deveres na sociedade, isso no âmbito social. Saúde é ela ter uma condição boa de trabalhar, dela conseguir prover condições para ela poder ter dinheiro para viajar, para comer, para estudar. Também envolve a parte fisiológica, ela está bem consigo mesma tanto psiquicamente quanto fisiologicamente. A saúde envolve tanto o contexto social, psíquico, quanto fisiológico. Saúde é a pessoa ter a capacidade de viver bem nesses três setores, nesses três meios, conseguindo ficar bem com ela mesma, nisso tudo. (E5 - gestor)

O interacionismo simbólico parte do pressuposto de que as ações dos indivíduos são orientadas de acordo com os seus próprios interesses, e a interação no mundo favorece a capacidade de (re)orientar suas ações (Blumer, 1969). Por isso, os profissionais da APS, diante das concepções de saúde, precisam desenvolver competência para utilizar o cotidiano enquanto problematizador. Assim, as interações profissionais devem ser nutridas com o conceito ampliado de saúde para superação das práticas ancoradas no modelo biomédico, ainda tão presentes nos serviços. 
A intersetorialidade não se opõe à ação setorial, mas remete ao sentido de complementariedade de práticas. Há necessidade de combinar a dimensão setorial com a intersetorial, pois, aos profissionais da APS, cabe atuar de forma interdisciplinar, multiprofissional, com responsabilidade integral sobre a população ao utilizar práticas de trabalho inovadoras e compartilhadas (Akerman, Franco, Moyses, Rezende, \& Rocha, 2014; Melo, Miranda, Silva, \& Limeira, 2018). Tarefas essas que, além da constituição de um sistema de qualidade, com acesso universal e integral, ampliam-se na direção de um papel articulador e integrador com outros setores, também determinantes das condições de vida e de saúde (Yasui et al., 2018), dimensões que podem ser apreciadas na fala:

Acho que é a junção, a conjugação dos setores, em todos os aspectos. Como a gente fala de saúde em um estado amplo, os setores da vida. . . também. . . seria a integração de todos os aspectos da vida, como um todo. A saúde integral, cada setor cooperando com o outro, se ajudando mutuamente. (E29 - ESF)

A compreensão da determinação social do processo saúde-doença, a dimensão do impacto de ações não especificamente setoriais sobre a saúde - tais como habitação, emprego e renda, cultura e lazer - e a impotência setorial diante da complexidade do mundo real e concreto fazem com que o setor saúde esteja mais mobilizado para propor articulações intersetoriais (Silva \& Tavares, 2016). Algumas percepções de profissionais das equipes de saúde sobre a intersetorialidade priorizam a integração e a complementariedade dos serviços, o que pode ser observado nas seguintes opiniões:

Essa ponte que a gente faz com outros equipamentos, não somente os equipamentos da saúde, mas também os equipamentos da assistência, da educação [. . .] Quando a gente pensa em olhar o sujeito num todo é importante olhar os outros equipamentos também, os outros setores, porque eles podem ser muito parceiros para gente conseguir realizar as nossas ações e promover a saúde das pessoas que a gente atende. (E21 - NASF-AB)

Intersetorialidade nós podemos entender que são vários serviços no território, no município, que atende às necessidades da população. (E58 - NASF-AB)

Em meio a essa discussão, torna-se necessário discutir a definição do termo interdisciplinaridade, já que, para os casos clínicos mais complexos, o trabalho compartilhado é um recurso de ampliação da atuação dos profissionais na direção de outras áreas do conhecimento (Matuda, Pinto, Martins, \& Frazão, 2015), e é por meio da interdisciplinaridade do cuidado que os vários olhares dos profissionais se encontram, para uma troca de saberes e o compartilhamento de estratégias de trabalho que busquem uma atenção ampliada e que possibilitem o cuidado do sujeito de acordo com suas necessidades e de forma integral (Bispo, Tavares, \& Tomaz, 2014). Alguns participantes confundiram o termo interdisciplinaridade com o termo intersetorialidade:

Intersetorialidade seria, a meu ver, essa capacidade dos profissionais se conectarem, eu acredito. Da equipe funcionar entrosada, de ter essa conexão profissional [ . . .]. (E18 - ESF)

É o setor onde todo mundo trabalha em conjunto [. . . ]. (E36 - ESF)

Vale ressaltar que os termos são complementares e que, entre as atribuições da APS, a intersetorialidade sugere aos trabalhadores da saúde ferramentas para ampliarem a 
integralidade do cuidado, responsabilização e resolutividade, construindo uma resposta mais eficaz para os problemas cotidianos. A intersetorialidade é um termo dotado de vários significados e possibilidades de aplicação prática, que se apoia no reconhecimento de que a relação entre "setores" implica mudanças importantes na ampliação da democracia e da cidadania (Pereira, 2014). Para a efetivação das práticas intersetoriais, os profissionais da ESF e NASF-AB, cuja atuação os coloca diante dos contextos de vida e saúde das pessoas, precisam compreender tanto os limites setoriais quanto as vantagens das ações integradas (Prado \& Santos, 2014).

Dada a necessidade de compreender a relação com os profissionais da saúde sobre a intersetorialidade nas políticas públicas, o Interacionismo Simbólico despontou como referencial teórico adequado. O Interacionismo Simbólico concebe o ser humano como ativo em sua vida, sob a influência dos significados estabelecidos em sua vivência. Os significados são elaborados, mantidos ou transformados na interação social, tanto consigo quanto com o outro. Ou seja, criam-se conceitos e age-se a partir deles, mas sob a influência desses no passado e futuro (Charon, 1985).

\section{Considerações Finais}

A definição sobre saúde trazida pelos participantes diz muito sobre a prática de saúde no cotidiano da APS de cada profissional, pois pode ser atribuída ao conceito ampliado ou, então, à concepção do modelo biomédico. Assim, com a saúde em seu conceito ampliado, há expansão das práticas para outras políticas públicas e/ou parcerias comunitárias. O modelo biomédico reduz a atuação meramente à ausência de doenças que restringem a atuação profissional à equipe ou, quando muito, à rede intrassetorial. Já a noção de intersetorialidade surge como estratégia de cuidado em rede, pois prioriza a integração, parceria, ação conjunta e união de todos os setores para a resolução de problemas comuns.

No Brasil, a intersetorialidade apresenta-se como essencial na promoção da saúde da população e da equidade em saúde. No entanto as políticas públicas urgem integração para alcançar os determinantes sociais de maneira coletiva, levando em consideração as vulnerabilidades, as necessidades e os arranjos locais. No cenário internacional, por recomendação da OMS, alguns países vêm trabalhando na perspectiva de integração das políticas públicas com base na abordagem Saúde em Todas as Políticas (Health in All Policies [HiAP]); experiências que podem nortear a realidade brasileira.

Por fim, vale ressaltar que a fundamentação da Teoria do Interacionismo Simbólico, enquanto ponto reflexivo, abre possibilidade para a ação interativa enquanto promoção do cuidado na APS, dada a sua perspectiva de compreensão dos aspectos subjetivos das pessoas. As problematizações trazidas no estudo podem produzir ressonância para atores da ESF e NASF-AB e contribuir para um maior entendimento do trabalho na APS, favorecendo a construção de políticas públicas viáveis de serem realistas no contexto. No âmbito local, é possível observar questões causadas por múltiplos fatores que requerem sincronia entre setores diversos na busca de soluções possíveis; a saúde bem corresponde sua influência pelos fatores sociais, econômicos e ambientais.

Pesquisas como esta podem expandir os horizontes para o conhecimento e a formação de profissionais, ampliando, assim, perspectivas que promovam um cuidar que conceba o 
indivíduo como um todo, abarcando os determinantes sociais da saúde por meio de ações intersetoriais. Há, ainda, de se lançar mão de discussão aprofundada da saúde como objeto de trabalho, principalmente com reflexões no cotidiano dos serviços.

\section{Referências}

Akerman, M., Franco, S. R., Moyses, S., Rezende, R., \& Rocha, D. (2014). Intersetorialidade? IntersetorialidadeS! Ciência e Saúde Coletiva [on-line], 19(11), 4291-4300. Recuperado de http://www.scielo.br/scielo.php?script=sci_arttext\&pid=S1413

Almeida, P. F., Medina, M. G., Fausto, M. C. R., Giovanella, L., Bousquat, A., \& Mendonça, M. H. M. (2018). Coordenação do cuidado e atenção primária à saúde no Sistema Único de Saúde. Saúde em Debate, 42(especial), 244-260. doi:https://dx.doi. org/10.1590/0103-11042018s116

Bardin, L. (2011). Análise de conteúdo. Lisboa, Portugal: Edições 70.

Barreto, M. L. (2017). Desigualdades em saúde: uma perspectiva global. Ciência \& Saúde Coletiva, 22(7), 2097-2108. doi:https://dx.doi.org/10.1590/1413-81232017227.02742017

Bispo, E. P. F., Tavares, C. H. F., \& Tomaz, J. M. T. (2014). Interdisciplinaridade no ensino em saúde: O olhar do preceptor na Saúde da Família. Interface-Comunicação, Saúde, Educação, 18(49), 337-350. [Epub March 10, 2014]. doi:https://dx.doi.org/10.1590/1807-57622013.0158

Blumer, H. (1969). Symbolic Interactionism: Perspective and Method. Berkeley: University of California Press.

Charon, M. (1985). Symbolic interactionism: An introduction, an interpretation, an integration. Califórnia: Editora Englewood Cliffs.

Czeresnia, D., Maciel, E. M. G. S, \& Oviedo, R. A. M. (2013). Os sentidos da saúde e da doença. Rio de Janeiro: Fiocruz.

Dubois, A., St-Pierre, L., \& Veras, M. (2015). A scoping review of definitions and frameworks of intersectoral action. Ciência \& Saúde Coletiva, 20(10), 2933-2942. doi:https://dx.doi. org/10.1590/1413-812320152010.01222014

Fertonani, H. P., Pires, D. E. P., Biff, D., \& Scherer, M. D. A. (2015). Modelo assistencial em saúde: Conceitos e desafios para a atenção básica brasileira. Ciência \& Saúde Coletiva, 20(6), 1869-1878. doi:https://dx.doi.org/10.1590/1413-81232015206.13272014

Fisher, M., Baum, F. E., MacDougall, C., Newman, L., McDermott, D., \& Phillips, C. (2017). Intersectoral action on SDH and equity in Australian health policy. Health Promotion International, 32(6), 1, 953-963. doi:https://doi.org/10.1093/heapro/daw035.

Garbois, J. A., Sodré, F., \& Dalbello-Araujo, M. (2017). Da noção de determinação social à de determinantes sociais da saúde. Saúde em Debate, 41(112), 63-76. doi:https://dx.doi. org/10.1590/0103-1104201711206

Lotta, G., \& Favareto, A. (2016). Desafios da integração nos novos arranjos institucionais de políticas públicas no Brasil. Sociologia Política [on-line], 24(57), 49-65. Recuperado de http://www.scielo.br/scielo.php?script=sci_arttext\&pid=S0104-.

Mattig T., Cantoreggi N., Simos J., Kruit C. F., \& Christie, D. P. T. H. (2017). HIA in Switzerland: Strategies for achieving Health in All Policies. Health Promotion International, 32(1), 149156. doi:https://doi.org/10.1093/heapro/dav087

Matuda, C. G., Pinto, N. R. S., Martins, C. L., \& Frazão, P. (2015). Colaboração interprofissional na Estratégia Saúde da Família: Implicações para a produção do cuidado e a 
gestão do trabalho. Ciência \& Saúde Coletiva, 20(8), 2511-2521. doi:https://dx.doi. org/10.1590/1413-81232015208.11652014

Melo, E. A., Miranda, L., Silva, A. M., \& Limeira, R. M. N. (2018). Dez anos dos Núcleos de Apoio à Saúde da Família (NASF): Problematizando alguns desafios. Saúde em Debate, 42(especial), 328-340. doi:https://dx.doi.org/10.1590/0103-11042018s12

Menezes, E. L. C., Scherer, M. D. A., Verdi, M. I., \& Pires, D. P. (2017). Modos de produzir cuidado e a universalidade do acesso na atenção primária à saúde. Saúde e Sociedade, 26(4), 888-903. doi:https://dx.doi.org/10.1590/s0104-12902017170497

Minayo, M. (2017). Amostragem e saturação em pesquisa qualitativa: Consensos e controvérsias. Revista Pesquisa Qualitativa, 5(7), 1-12. Recuperado de https://editora. sepq.org.br/index.php/rpq/article/view/82/59

Molina, J. (2018). Saúde universal com equidade, sem deixar ninguém para trás. Revista Panamericana de Salud Pública [on-line]. 42, e173. doi:https://doi.org/10.26633/ RPSP.2018.173

Pereira, P. A. P. (2014). A intersetorialidade das políticas sociais na perspectiva dialética. In Monnerat, G. L., Almeida, N. L. T., Souza, R. G. de (Orgs.), A intersetorialidade na agenda das políticas sociais (pp. 21-39). Campinas: Papel Social.

Prado, N. M. B. L., \& Santos, A. M. (2018). Promoção da saúde na Atenção Primária à Saúde: Sistematização de desafios e estratégias intersetoriais. Saúde em Debate, 42(especial), 379-395. doi:https://dx.doi.org/10.1590/0103-11042018s126

Shankardass, K., Muntaner, C., Kokkinen, L., Shahidi, F. V., Freiler, A., Oneka, G. . . Patricia O'Campo (2018). The implementation of health in all policies initiatives: A systems framework for government action. Health Research Policy and Systems. 16(1). 26. doi:https://doi.org/10.1186/s12961-018-0295-z

Silva, D. A. J., Tavares, M. F. (2016). Ação intersetorial: Potencialidades e dificuldades do trabalho em equipes da Estratégia Saúde da Família na cidade do Rio de Janeiro. Saúde em Debate, 40(111), 193-205. doi:https://dx.doi.org/10.1590/0103-1104201611115

Souza, S., Costa, R., Shiroma, L., Maliska, I. C., Amadigi, F., Pires, D., \& Ramos, F. (2010). Reflexões de profissionais de saúde acerca do seu processo de trabalho. Revista Eletrônica de Enfermagem, 12(3), 449-455. doi:https://doi.org/10.5216/ree.v12i3.6855

Tesser, C. D. (2017). Núcleos de Apoio à Saúde da Família, seus potenciais e entraves: Uma interpretação a partir da atenção primária à saúde. Interface - Comunicação, Saúde, Educação, 21(62), 565-578. [Epub, 3 de novembro de 2016]. doi:https://dx.doi. org/10.1590/1807-57622015.0939

United Nations. (2015). A/70/1. Transforming our world: The 2030 Agenda for Sustainable Development. Nova lorque: UN. Recuperado de www.un.org/ga/search/view_doc. asp?symbol=A/RES/70/1\&Lang=E

Yasui, S., Luzio, C., \& Amarante, P. (2018). Atenção psicossocial e atenção básica: A vida como ela é no território. Revista Polis e Psique, 8(1), 173-190.

Recebido em: 08/08/2019

Última revisão: 09/12/2019

Aceite final: 11/02/2020 


\section{Sobre as autoras:}

Edna Mara Mendonça: Doutoranda em Saúde Coletiva no Instituto René Rachou - Fiocruz Minas. Mestre em Ciências pela Universidade Federal de São João Del-Rei (UFSJ). Especialista em Atenção ao Usuário de Drogas no Sistema Único de Saúde (SUS) pela Escola de Saúde Pública de Minas Gerais (ESPMG) e Gestão de Redes de Atenção à Saúde pela Escola Nacional de Saúde Pública Sérgio Arouca (ENSP). Terapeuta Ocupacional com atuação nos seguintes temas: saúde mental, álcool e outras drogas, atenção primária à saúde, articulação intersetorial, educação permanente, apoio matricial, violência contra as mulheres, rede socioassistencial e territórios de alta vulnerabilidade social. E-mail: ednamaras@gmail.com, Orcid: http://orcid.org/0000-0003-4256-1407

Fernanda Moura Lanza: Doutora em Enfermagem. Mestre em Saúde e Enfermagem pela Universidade Federal de Minas Gerais (UFMG). Especialista em Enfermagem Hospitalar- Neonatologia. Graduação em Enfermagem pela UFMG. Professora adjunta III do Curso de Enfermagem na Universidade Federal de São João Del-Rei (UFSJ), Campus Centro-Oeste Dona Lindu (CCO), Grupo de Atuação Docente Saúde Coletiva. Docente do Programa de Pós-Graduação Mestrado Acadêmico em Enfermagem da UFSJ/CCO, na linha de pesquisa Gestão em Serviços de Saúde e Enfermagem. Docente do Programa de Residência em Enfermagem na Atenção Básica/Saúde da Família da UFSJ/CCO. Membro do Núcleo de Estudos e Pesquisas em Avaliação e Gestão em Saúde (NEPAG) da UFSJ/CCO; do Núcleo de Estudos e Pesquisas em Hanseníase (NEPHANS) da Escola de Enfermagem da Universidade Federal de Minas Gerais (EEUFMG); e da Comissão de Prevenção e Tratamento de Lesões Cutâneas da Secretaria Municipal de Saúde de Divinópolis, MG. E-mail: fernandalanza@ufsj.edu.br, Orcid: http://orcid.org/0000-0001-8250-180X 